Check for updates

Cite this: Chem. Sci., 2019, 10, 9038

๑ All publication charges for this article have been paid for by the Royal Society of Chemistry

Received 21st June 2019

Accepted 8th August 2019

DOI: $10.1039 /$ c9sc03061a

rsc.li/chemical-science

\section{Two-step synthesis of a red-emissive warped nanographene derivative via a ten-fold $\mathrm{C}-\mathrm{H}$ borylation $\uparrow$}

\author{
Kenta Kato, ${ }^{a}$ Hsing-An Lin, ${ }^{\text {ab }}$ Motonobu Kuwayama, abc Mai Nagase, ${ }^{a}$ \\ Yasutomo Segawa, (D) *ab Lawrence T. Scott ${ }^{\mathrm{d}}$ and Kenichiro Itami (D) *abc
}

The regioselective ten-fold borylation of warped nanographene (WNG: $\mathrm{C}_{80} \mathrm{H}_{30}$ ) was achieved by modifying the reaction conditions of a previously reported Ir-catalyzed $\mathrm{C}-\mathrm{H}$ borylation, affording decaborylated WNG in high yield (75\%) from pristine WNG. The solid-state structure of decaborylated WNG was confirmed by Xray crystallography. Corresponding decaarylated WNGs containing electron-withdrawing and -donating groups were synthesized from decaborylated WNG using Suzuki-Miyaura cross-coupling reactions to afford the red-emissive warped nanographene derivative.

\section{Introduction}

Negatively curved nanographenes are attracting much attention as a new form of carbon. ${ }^{1} \mathrm{sp}^{2}$-hybridized carbons form graphene and carbon nanotubes by constructing a hexagonal lattice. ${ }^{2}$ When a small ring such as a five-membered ring or a four-membered ring is incorporated into this structure, it becomes a bowl-shaped structure with a positive curvature resulting in a carbon nanotube endcap or fullerenes. ${ }^{3,4}$ On the other hand, if a relatively large ring, a seven- or an eightmembered ring, is introduced, the carbon sheet forms a saddle shape having a negative curvature. ${ }^{4}$ It is well known that carbon structures can be extended three-dimensionally by introducing negative curvatures, and various kinds of exotic nanocarbons, such as carbon nanotube joints, saddle-shaped sheets, Mackay crystals, carbon coils, and carbon tori, have been proposed. ${ }^{4,5}$ However, because there was no effective way to synthesize these negatively curved nanocarbons, research on their synthesis and function has not been developed. In 2013, we reported the synthesis of warped nanographene (WNG), a grossly warped polycyclic aromatic hydrocarbon (PAH) with five seven-membered rings (Fig. 1). WNG has different properties from planar PAHs. ${ }^{6}$ For example, WNG can be dissolved in organic solvents because the van der Waals interaction between

${ }^{a}$ Graduate School of Science, Nagoya University, Chikusa, Nagoya, 464-8602, Japan. E-mail:Itami@chem.nagoya-u.ac.jp; ysegawa@nagoya-u.jp

${ }^{b} J S T-E R A T O$, Itami Molecular Nanocarbon Project, Nagoya University, Chikusa, Nagoya, 464-8602, Japan

'Institute of Transformative Bio-Molecules (WPI-ITbM), Nagoya University, Chikusa, Nagoya, 464-8602, Japan

${ }^{d}$ Department of Chemistry, University of Nevada, Reno, NV 89557-0216, USA

$\dagger$ Electronic supplementary information (ESI) available. CCDC 1893066. For ESI and crystallographic data in CIF or other electronic format see DOI: 10.1039/c9sc03061a
WNG molecules is small due to the curved structure. In general, big and planar PAHs cannot be dissolved in organic solvents, ${ }^{7}$ and even hexabenzocoronene, which is smaller than WNG, must be substituted to enable solution-state transformations, ${ }^{8}$ except for perchlorination. ${ }^{9}$

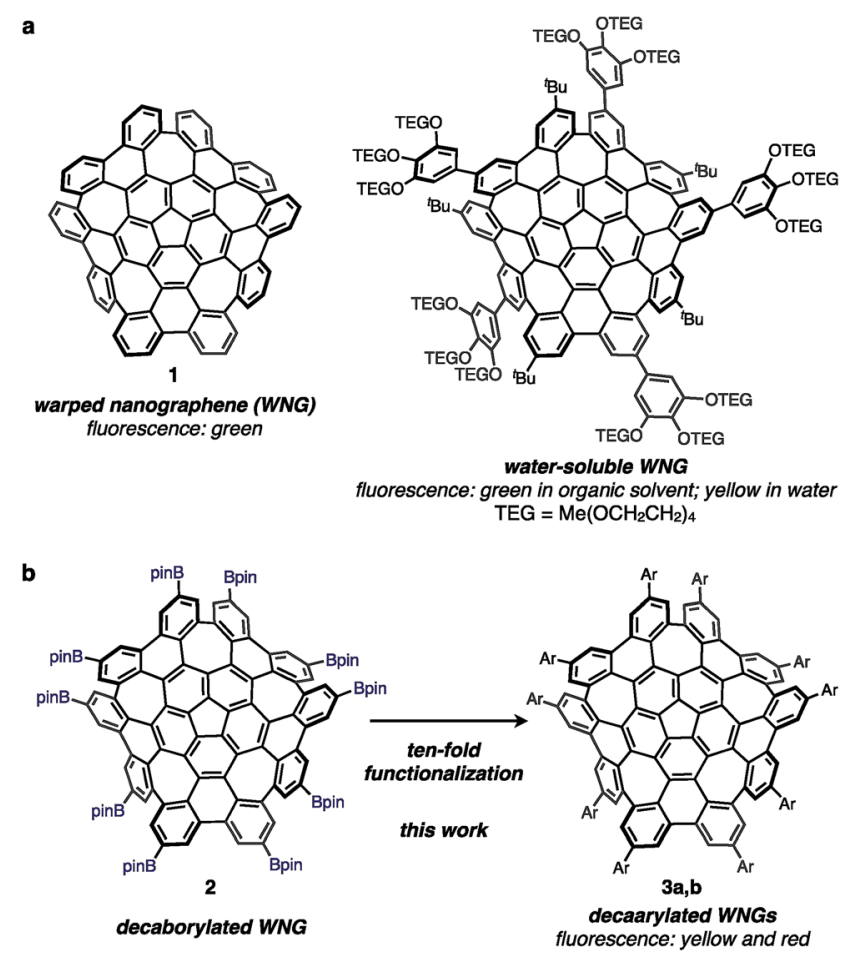

Fig. 1 Warped nanographene (WNG) and its derivatives. (a) Structures of WNG and water-soluble WNG; (b) ten-fold functionalization of WNG via decaborylated WNG (this work). 
Furthermore, WNG exhibits green fluorescence in organic solvents and water. Using these properties, we succeeded in the synthesis of water-soluble WNG and its application to bioimaging in 2018 (Fig. 1)..$^{10}$ Water-soluble WNG accumulates in lysosomes of HeLa cells and acts as a fluorescent probe. In addition, HeLa cells incorporating water-soluble WNG can be selectively killed by visible light irradiation. This is because water-soluble WNG is not cytotoxic and has the ability to generate singlet oxygen. Thus, the usefulness of highly curved nanographene has been demonstrated. However, all of the WNGs synthesized so far are green fluorescent and have not been successfully tuned electronically. ${ }^{6,10}$ In particular, long wavelength absorption and fluorescence are essential for the applications of chemical biology. Since pristine WNG is currently commercially available, ${ }^{11}$ electronically tuned WNG can be easily obtained if a method for functionalizing pristine WNG is developed.

Herein we report the rapid synthesis of WNG derivatives using iridium-catalyzed decaborylation as a key reaction (Fig. 1). The iridium-catalyzed borylation of PAHs is a powerful reaction to functionalize aromatic rings with high selectivity. ${ }^{12}$ Because the boryl group is a platform that can be converted into various functional groups, ${ }^{13}$ it is expected that a variety of WNG derivatives can be easily obtained via this method.

\section{Results and discussion}

\section{Synthesis and structure}

Initially, we screened the reaction conditions (e.g. solvents, temperature, and ligands) of this $\mathrm{C}-\mathrm{H}$ borylation in order to find suitable conditions for the ten-fold $\mathrm{C}-\mathrm{H}$ borylation reaction of 1. Rather than $4,4^{\prime}$-di-tert-butyl-2,2'-bipyridyl, which is a ligand conventionally used for such $\mathrm{C}-\mathrm{H}$ borylations, we selected 3,4,7,8-tetramethyl-1,10-phenanthroline (tmphen) to increase the catalytic reactivity. ${ }^{14}$ THF, mesitylene (1,3,5-trimetylbenzene), and alkanes proved to be unsuitable solvents. Finally, cyclopentyl methyl ether (CPME) was identified as a suitable solvent, and we successfully obtained the decaborylated WNG 2 in 75\% isolated yield when 1 and HBpin (Bpin = 4,4,5,5-tetramethyl-1,3,2-dioxaborolan-2-yl) were stirred at $120^{\circ} \mathrm{C}$ in a sealed tube in the presence of a catalytic amount of $[\mathrm{Ir}(\mathrm{OMe}) \operatorname{cod}]_{2}(25 \mathrm{~mol} \%)$ and tmphen (50 mol\%) (Fig. 2a). Ten borylation is the largest among previously reported $\mathrm{C}-\mathrm{H}$ borylation of pristine PAHs such as pyrene, perylene, coronene, and corannulene. $^{15,16}$ The structure of 2 was identified on the basis of ${ }^{1} \mathrm{H}$ and ${ }^{13} \mathrm{C}$ NMR spectroscopy as well as high-resolution mass spectrometry measurements. In the ${ }^{1} \mathrm{H}$ NMR spectrum of $\mathbf{4}$ in $\mathrm{C}_{2} \mathrm{D}_{2} \mathrm{Cl}_{4}$ at $140{ }^{\circ} \mathrm{C}$, three singlets with a $1: 1: 12$ integration ratio were observed at $9.10,7.87$ and $1.48 \mathrm{ppm}$, respectively, which indicates that 2 exhibits ten-fold pseudosymmetry in solution due to rapid dynamic motion at such high temperatures. This observation is consistent with the behavior of previously reported WNG derivatives. ${ }^{6,10}$

The molecular structure of 2 was further confirmed by singlecrystal X-ray diffraction analysis (Fig. $2 \mathrm{~b}$ and c). Suitable crystals were obtained from the slow evaporation of a saturated diethyl ether solution of 2. The X-ray crystal structure analysis confirmed our expectation that the $\mathrm{C}-\mathrm{H}$ borylation of $\mathbf{1}$ occurs at sterically unhindered positions. The core structure of 2 is similar to those of previously reported WNG derivatives, ${ }^{6,10}$

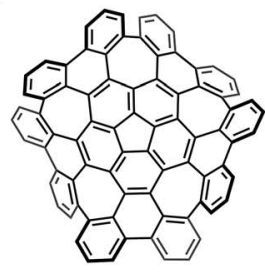

1

d

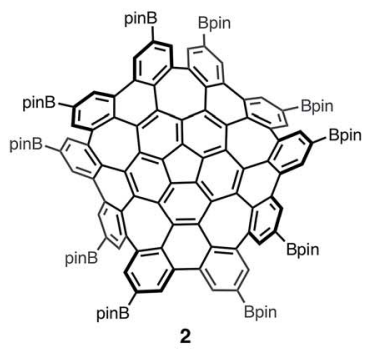

HBpin (30 equiv) $[\mathrm{lr}(\mathrm{OMe}) \mathrm{cod}]_{2}(25 \mathrm{~mol} \%)$ tmphen (50 mol\%) CPME $120^{\circ} \mathrm{C}, 24 \mathrm{~h}$
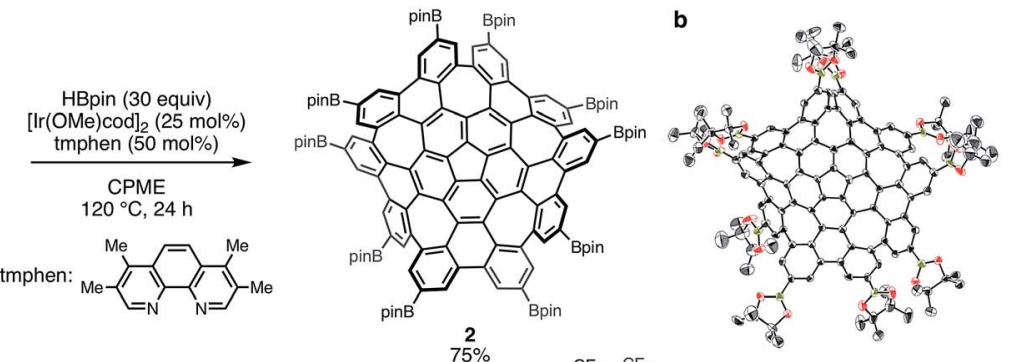

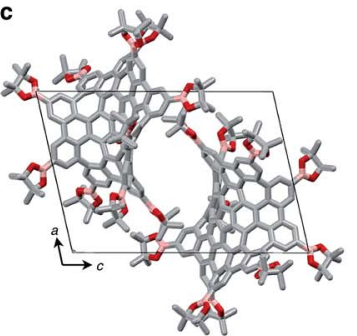

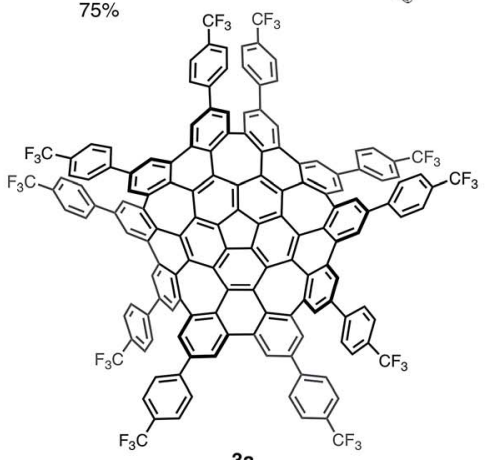

$3 a$
$60 \%$

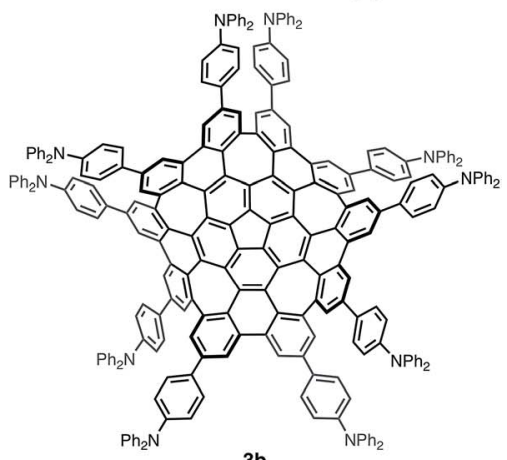

$3 b$
$58 \%$

Fig. 2 Synthesis of decaarylated WNGs via the ten-fold C-H borylation of WNG. (a) Ten-fold C-H borylation of 1. (b) ORTEP drawing of 2 with thermal ellipsoids set to 50\% probability; all hydrogen atoms and solvent molecules are omitted for clarity; gray: carbon; yellow: boron; red: oxygen. (c) Packing mode of 2 viewed along the $b$ axis. (d) Suzuki-Miyaura cross-coupling of 2 with aryl halides $4 a$, $b$ to give decaarylated WNGs 3a,b. Abbreviations: Bpin = 4,4,5,5-tetramethyl-1,3,2-dioxaborolan-2-yl; cod =1,5-cyclooctadiene; CPME = cyclopentyl methyl ether; dba = dibenzylideneacetone; SPhos $=2$-dicyclohexylphosphino-2', $6^{\prime}$-dimethoxybiphenyl. 
which indicates that the effect of the ten boryl groups on the warped structure of the WNG core is negligible. The packing structure of 2, viewed along the $b$ axis, is shown in Fig. 2c. Due to the bulky boryl groups, $\pi-\pi$ interactions between WNG cores could not be observed, which is in good agreement with the high solubility of 2 in organic solvents. Co-crystallized $\mathrm{Et}_{2} \mathrm{O}$ molecules are contained within the cavities.

With the decaborylated WNG (2) in hand, we then attempted to engage 2 in Suzuki-Miyaura cross-coupling reactions in order to demonstrate the potential utility of 2 as a universal synthetic platform for the rapid preparation of WNG derivatives. As coupling partners, $p$-(trifluoromethyl)phenyl and $p$-(diphenylamino)phenyl groups were selected as electron-withdrawing and -donating groups, respectively, with the expectation to modify the electronic properties of WNG. The ten-fold Suzuki-Miyaura cross-coupling reactions of 2 with the corresponding aryl halides $(\mathbf{4 a}, \mathbf{b})$ were carried out for $3 \mathrm{~h}$ at $100{ }^{\circ} \mathrm{C}$ in toluene/water in the presence of $\mathrm{Pd}_{2}(\mathrm{dba})_{3} \cdot \mathrm{CHCl}_{3}, 2$-dicyclohexylphosphino$2^{\prime}, 6^{\prime}$-dimethoxybiphenyl (SPhos) and $\mathrm{Cs}_{2} \mathrm{CO}_{3}$. The corresponding decaarylated WNGs (3a,b) with electron-withdrawing and -donating groups were obtained in $60 \%$ and $58 \%$ yield, respectively. The structures of $\mathbf{3 a}, \mathbf{b}$ were assigned on the basis of high-resolution mass spectrometry, as the ${ }^{1} \mathrm{H}$ and ${ }^{13} \mathrm{C}$ NMR signals of $\mathbf{3 a}, \mathbf{b}$ were broadened even at $100^{\circ} \mathrm{C}$. The singlet signal observed in the ${ }^{19} \mathrm{~F}$ NMR spectrum of $3 \mathbf{a}(-63.6 \mathrm{ppm})$ supports the attributed pseudosymmetric structure in solution at $100{ }^{\circ} \mathrm{C}$. Notably, 3a is soluble not only in non-polar organic solvents but also in polar solvents such as acetone and methanol.

\section{Photophysical properties}

To gain further insight into the effect of the presence of peripheral functional groups on the photophysical properties of the WNG core, the UV-vis absorption spectra, fluorescence spectra and fluorescence quantum yields of $\mathbf{2}, \mathbf{3 a}$, and $\mathbf{3 b}$ were measured and compared with those of $\mathbf{1}$ (Fig. 3a). The absorption and fluorescence spectra of 1 reveal characteristic peaks at $418 \mathrm{~nm}$ and $500 \mathrm{~nm}$, respectively. Both the absorption and fluorescence spectra of 2 are similar in shape to the corresponding spectra of $\mathbf{1}$, albeit slightly shifted bathochromically (434 $\mathrm{nm}$ and $510 \mathrm{~nm}$ ). In the case of $3 \mathbf{a}$ and $\mathbf{3 b}$, the spectra are broadened and also bathochromically shifted. This red-shift is also reflected in the fluorescence color, which changes from green (1 and 2) to yellow (3a) and red (3b). It is worth noting that the quantum yield of $\mathbf{3 b}(0.47)$ is higher than those of $\mathbf{1}(0.26), 2$ (0.20) and $3 \mathrm{a}(0.28)$. Time-dependent density functional theory (TD-DFT) calculations at the B3LYP/6-31G(d) level of theory were used to determine the shape, energy levels and potential transitions between the HOMO and LUMO of 1, 2, 3a and $\mathbf{3 b}$ (Fig. 3b). The results of these calculations suggest that the introduction of peripheral substituents affects the energy levels of the HOMOs and LUMOs in $\mathbf{2}, \mathbf{3} \mathbf{a}$ and $\mathbf{3 b}$ relative to those of $\mathbf{1}$. In $2, \mathbf{3 a}$ and $\mathbf{3 b}$, the shapes of the HOMOs and LUMOs are very similar to those of $\mathbf{1}$ except for the HOMO of $\mathbf{3 b}$, in which effective $\pi$-conjugation is observed between the WNG core and the aminophenyl groups. As expected, the electron-withdrawing trifluoromethylphenyl groups of 3a lower the energy level of its HOMO and LUMO, while the electron-donating diphenylaminophenyl groups increase the energy level of the HOMO of $\mathbf{3 b}$. The HOMO-LUMO energy gap decreases in the order $1(3.30 \mathrm{eV})$ $>2(2.88 \mathrm{eV})>3 \mathbf{a}(2.80 \mathrm{eV})>3 \mathbf{b}(2.62 \mathrm{eV})$, which is in good agreement with the bathochromic shifts observed in the absorption and fluorescence spectra. According to the TD-DFT calculations, the HOMO-LUMO transitions are symmetrically allowed, and, most notably, the oscillator strength of $\mathbf{3 b}(f=$ 0.321) is higher than those of the other three compounds, probably due to the $\pi$-conjugation effect. The increase of the oscillator strength might be responsible for the higher quantum yield of $\mathbf{3 b}$ relative to that of $\mathbf{1}$, despite the fact that the fluorescence of $\mathbf{3 b}$ is bathochromically shifted relative to that of $\mathbf{1}$.
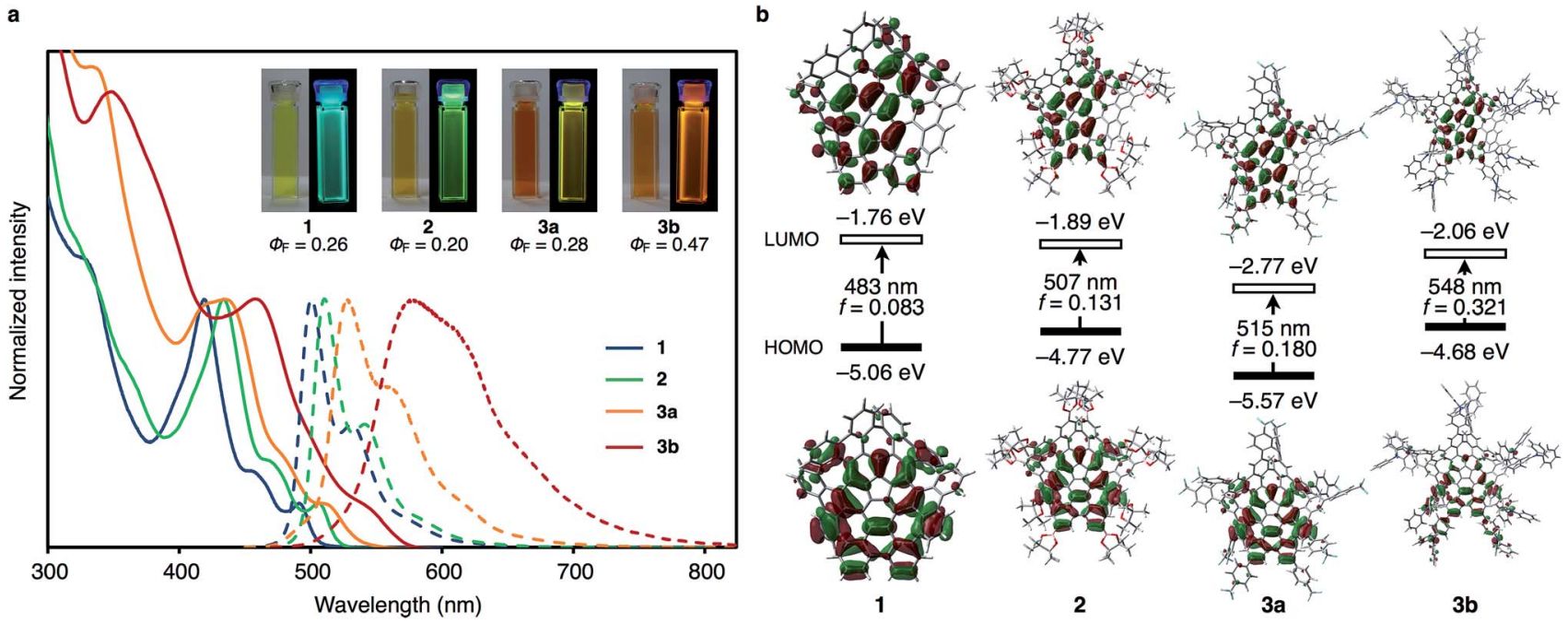

Fig. 3 Photophysical properties of WNG derivatives. (a) UV/vis absorption (solid lines) spectra, fluorescence (dotted lines) spectra, absolute fluorescence quantum yields $\left(\Phi_{\mathrm{F}}\right)$, and photographs of dichloromethane solutions of $1,2,3 \mathrm{a}$, and $3 \mathrm{~b}$ recorded under illumination with either ambient or UV light (254 nm). (b) Frontier molecular orbitals and energy levels of 1, 2, 3a, and 3b calculated at the B3LYP/6-31G(d) level of theory. Excitation energies were calculated by TD-DFT calculations at the same level. The $f$ values refer to the oscillator strength. 


\section{Conclusions}

We have developed a method for the ten-fold borylation of pristine WNG $\left(\mathrm{C}_{80} \mathrm{H}_{30}\right)$, which dramatically increases the solubility of WNG compared to other planar PAHs of similar size. This is the first report on the $\mathrm{C}-\mathrm{H}$ borylation of non-substituted PAHs larger than coronene $\left(\mathrm{C}_{24} \mathrm{H}_{12}\right)$. The transition-metalcatalyzed functionalization of such large PAHs without any solubilizing substituents was enabled by the grossly warped structure of 1. Moreover, we confirmed that the thus-obtained decaborylated WNG (2) is a useful synthetic intermediate for the generation of functionalized WNGs. In order to demonstrate the feasibility of this concept, we engaged 2 in SuzukiMiyaura cross-coupling reactions with aryl halides (4a,b), which provided decaarylated WNGs in high yields. The introduction of electron-withdrawing or -donating groups effectively tunes the photophysical properties of WNG, which is reflected in the bathochromically shifted fluorescence with a higher quantum yield of $\mathbf{3 b}$ relative to that of $\mathbf{1}$. This behavior can be explained in terms of an efficient $\pi$-conjugation of the WNG core and the peripheral aminophenyl groups. The electron withdrawing aryl groups in 3a lower the LUMO by $1.0 \mathrm{eV}$, and the electrondonating aryl groups in $\mathbf{3 b}$ raise the HOMO by $0.4 \mathrm{eV}$, relative to those frontier MOs in non-substituted 1. Given that pristine WNG is now commercially available, its derivatization via this borylation method as a key step can be expected to significantly accelerate the application of WNG-based functional materials.

\section{Conflicts of interest}

There are no conflicts to declare.

\section{Acknowledgements}

This work was supported by the ERATO program from JST (JPMJER1302 to K. I.), the Funding Program for KAKENHI from MEXT (JP19H05463 to K. I. and JP16K05771 and JP19H02701 to Y. S.), a Grant-in-Aid for Scientific Research on Innovative Areas " $\pi$-Figuration" from JSPS (JP17H05149 to Y. S.), the Noguchi Institute (to Y. S.) and the US National Science Foundation (CHE-1149096 to L. T. S.). K. K. acknowledges the IGER Program in Green Natural Sciences, Nagoya University and the JSPS fellowship for young scientists. Calculations were performed using the resources of the Research Center for Computational Science, Okazaki, Japan. ITbM is supported by the World Premier International Research Center Initiative (WPI), Japan.

\section{Notes and references}

1 (a) R. G. Harvey, Polycyclic Aromatic Hydrocarbons, WileyVCH, New York, 1997; (b) R. A. Pascal Jr, Chem. Rev., 2006, 106, 4809; (c) Y.-T. Wu and J. S. Siegel, Chem. Rev., 2006,
106, 4843; (d) V. M. Tsefrikas and L. T. Scott, Chem. Rev., 2006, 106, 4868; (e) H. Dodziuk, Strained Hydrocarbons: Beyond the van't Hoff and Le Bel Hypothesis, Wiley-VCH, Weinheim, Germany, 2009; (f) R. Rieger and K. Müllen, $J$. Phys. Org. Chem., 2010, 23, 315; $(g)$ M. A. Petrukhina and L. T. Scott, Fragments of Fullerenes and Carbon Nanotubes: Designed Synthesis, Unusual Reactions, and Coordination Chemistry, Wiley, 2011; (h) Y. Segawa, H. Ito and K. Itami, Nat. Rev. Mater., 2016, 1, 15002.

2 (a) S. Iijima, Nature, 1991, 354, 56; (b) K. S. Novoselov, A. K. Geim, S. V. Morozov, D. Jiang, Y. Zhang, S. V. Dubonos, I. V. Grigorieva and A. A. Firsov, Science, 2004, 306, 666.

3 H. W. Kroto, J. R. Heath, S. C. O'Brien, R. F. Curl and R. E. Smalley, Nature, 1985, 318, 162.

4 (a) S. Iijima, T. Ichihashi and Y. Ando, Nature, 1992, 356, 776; (b) S. H. Pun and Q. Miao, Acc. Chem. Res., 2018, 51, 1630.

5 (a) A. L. Mackay and H. Terrones, Nature, 1991, 352, 762; (b) D. N. Weldon, W. J. Blau and H. W. Zandbergen, Chem. Phys. Lett., 1995, 241, 365; (c) A. Takashima, T. Nishii and J. Onoe, J. Phys. D: Appl. Phys., 2012, 45, 485302.

6 (a) K. Kawasumi, Q. Zhang, Y. Segawa, L. T. Scott and K. Itami, Nat. Chem., 2013, 5, 739; (b) K. Kato, Y. Segawa, L. T. Scott and K. Itami, Chem.-Asian J., 2015, 10, 1635; (c) H.-A. Lin, K. Kato, Y. Segawa, L. T. Scott and K. Itami, Chem. Sci., 2019, 10, 2326; (d) J. I. Urgel, M. Di Giovannantonio, Y. Segawa, P. Ruffieux, L. T. Scott, C. A. Pignedoli, K. Itami and R. Fasel, J. Am. Chem. Soc., 2019, DOI: 10.1021/jacs.9b05501.

7 J. C. Fetzer Large Polycyclic Aromatic Molecules - Chemistry and Analysis, Wiley Interscience, New York, 2000.

8 R. Yamaguchi, S. Hiroto and H. Shinokubo, Org. Lett., 2012, 14, 2472.

9 Y.-Z. Tan, B. Yang, K. Parvez, A. Narita, S. Osella, D. Beljonne, X. Feng and K. Müllen, Nat. Commun., 2013, 4, 2646.

10 H. A. Lin, Y. Sato, Y. Segawa, T. Nishihara, N. Sugimoto, L. T. Scott, T. Higashiyama and K. Itami, Angew. Chem., Int. Ed., 2018, 57, 2874.

11 Kanto Chemical Co.Inc., catalog no. 45043-65.

12 T. Ishiyama, J. Takagi, K. Ishida, N. Miyaura, N. R. Anastasi and J. F. Hartwig, J. Am. Chem. Soc., 2002, 124, 390.

13 J. F. Hartwig, Acc. Chem. Res., 2012, 45, 864.

14 S. M. Preshlock, B. Ghaffari, P. E. Maligres, S. W. Krska, R. E. Maleczka and M. R. Smith, J. Am. Chem. Soc., 2013, 135, 7572.

15 D. N. Coventry, A. S. Batsanov, A. E. Goeta, J. A. K. Howard, T. B. Marder and R. N. Perutz, Chem. Commun., 2005, 41, 2172.

16 M. N. Eliseeva and L. T. Scott, J. Am. Chem. Soc., 2012, 134, 15169. 